\title{
ESR1 Status by Immunohistochemistry
}

National Cancer Institute

\section{Source}

National Cancer Institute. ESR1 Status by Immunohistochemistry. NCI Thesaurus. Code C157196.

An indication that expression or absence of ESR1 in a sample was determined using immunohistochemical staining techniques. 